\title{
Isothermal dehydration of thin films
}

\section{Calorimetric assessment of model parameters}

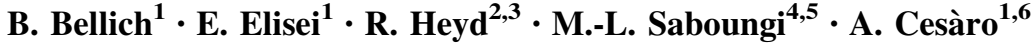

\begin{abstract}
The isothermal dehydration process was investigated by calorimetric experiments on water samples, pointing out a substantial correspondence with gravimetric results, even though the heat flow measurement is characterized by a higher resolution on approaching the end of the evaporation. The role of the geometry of the sample on water evaporation rate is analyzed with reference to two model geometries, resembling the spherical and the planar surface. Specific attention was given to the planar surface for the description of the water evaporation from thin film. The development of a theoretical model, which provides information regarding the water activity, was supported by investigating the effect of the several parameters involved in the isothermal dehydration, such as sample mass and temperature. The data collected revealed some limits in the valuable use of the theoretical model when the experimental conditions are outside the range of quasi-equilibrium
\end{abstract}

B. Bellich

bbellich@units.it

1 Department of Chemical and Pharmaceutical Sciences, University of Trieste, Via Giorgieri 1, 34127 Trieste, Italy

2 Interface, Confinement, Materials and Nanostructures (ICMN), CNRS/Orleans University, 1b rue de la Férollerie, 45071 Orléans Cedex 2, France

3 National Engineering School of Bizerta (ENIB), University of Cartage, BP66, Campus Universitaire Menzel Abderrahman, 7035 Bizerte, Tunisia

4 University of Orleans, Orleans, France

5 IMPMC, Sorbonne Universités-UPMC, CNRS UMR 7590, Muséum National d'Histoire Naturelle, IRD UMR 206, Paris, France

6 Elettra Sincrotrone Trieste, Area Science Park, 34149 Trieste, Italy thermodynamic condition, e.g., at temperature higher that $50{ }^{\circ} \mathrm{C}$. Attention has also been given to control the initial thermal unbalance in order to improve the measurements.

Keywords Water - Thin film - Isothermal dehydration . DSC - Mass and temperature dependence

\section{Introduction}

Water removal (dehydration) and uptake (adsorption or rehydration) involves interesting fundamental principles of physics and is of paramount relevance in several technological applications ranging from food, arts, medicine and cosmetics, not to speak about biology, chemistry, physics and geophysics. Under equilibrium or quasi-equilibrium conditions, the fundamental thermodynamic equations of liquid-vapor phase diagram must hold for pure liquids. From kinetic viewpoint, even for complex systems, when the diffusion of evaporation species from the bulk to the evaporation surface is sufficiently rapid, i.e., the concentration on the surface is the same as that in the bulk, and the evaporation rate is essentially controlled by the surface process.

Essentially, the mechanism for the surface process is that of monomolecular sporadic evaporation. For the sporadic process, the evaporation more or less slowly proceeds layer by layer; the molecular species are removed from one layer first and proceed to another layer only after the evaporation of the previous layer is complete. There may be a sufficient time for the evaporating species to diffuse from a bulk solution to the surface, and under these circumstances, the evaporating rate is still proportional to the surface area [1]. However, in most practical cases, the drying kinetics of complex materials depends on several mechanisms such as diffusion, convection, Marangoni effects and contact line 
motion, covering several scales from microscopic to macroscopic [1-6]. Polymeric formulations for cosmetics are an example of the former and plant and food systems of the latter [7-9]. Although the kinetics may involve different mechanisms, they share crucial key parameters such as the activity of water and its diffusivity through the matrix, the initial chemical composition having a strong impact on the morphology of the final dry product $[10,11]$.

In a previous paper [12], after the presentation of a theoretical model for a drying process in a DSC operating under isothermal conditions, the heuristic behavior of water dehydration from a thin film was illustrated giving attention to the role of sugar type and concentration. What appeared to be an extremely difficult problem was the quantitative comparison of theory with experiment in order to describe more complex dehydration processes by determining the value of the various parameters.

The approach presented needs to be fully validated by recognizing the constraints on the value of the parameters, so that various drying processes can be studied without recourse to special theories to be constructed and to additional ad hoc parameters. In this work, we shall consider the theory developed and provide the quantification of the underlying parameters as well the range of validity of the theory itself. In general, the mass transport of water from liquid surfaces is influenced by the temperature, gas phase composition, surface water activity, the bulk system rheological properties and the kinetics of transport through the surface region, but also by the geometrical experimental setup [13]. Additionally, the flux of mass from a surface requires the removal of latent heat from the surface through conduction and convection of heat within the surrounding gas phase [14].

In this study, firstly, two model systems characterized by different geometries, such as the drop and the film, are compared by gravimetric and calorimetric methods; then, the effect of the other operational parameters on the calorimetric output is explored, including temperature and sample mass. The final goal is to provide a more firm basis for the investigation of the evaporation of water from a film of complex composition from a kinetic and thermodynamic point of view.

\section{Materials and methods}

\section{Materials}

The following materials were used to carry out both the calorimetric and the gravimetric experiments: purified MilliQ water (Millipore Corporation, Bedford, MA) and filter paper (grade 93, particle retention $10 \mu \mathrm{m}$, Whatman International Ltd., UK).

\section{Gravimetric measurements}

The samples were analyzed with an electronic microbalance (Mettler Toledo XS205 Dual Range), and the data were recorded by a computer. All the measurements were taken at $20{ }^{\circ} \mathrm{C}$ in an environment with $\mathrm{RH}=40 \%$. The data analysis was performed with the software Origin ${ }^{\circledR} 8.0$.

\section{Calorimetric measurements}

Water evaporation was investigated with a heat flow (HF) calorimeter (DSC 6, PerkinElmer Instruments, Waltham, MA). Open aluminum pans (volume $40 \mu \mathrm{L}$ ) were used. The initial mass of water was measured with an electronic microbalance (Mettler Toledo XS205 Dual Range). The data collection was carried out with the software Pyris (2000 PerkinElmer Instruments LLC version 3.8), while the data analysis was made through the software Origin ${ }^{\circledR}$ 8.0. Unless otherwise reported, standard measurements were taken under isothermal conditions at $20^{\circ} \mathrm{C}$ and nitrogen flux set at $15 \mathrm{~mL} \mathrm{~min}^{-1}$.

\section{Results and discussion}

The main aim of this work was to fully investigate the parameters affecting water evaporation by using a microcalorimeter in isothermal conditions. To begin with, a summary of the main theoretical concepts developed in a previous paper [12] is presented, and then a series of topics is treated. The setup of the adequate experimental conditions required to consider firstly the effect of the paper as a support for a planar geometry. Within this frame, a comparison between gravimetric and calorimetric measurement is reported, giving attention also to the geometry of the sample. Secondly, the nitrogen flux, which provides a controlled atmosphere, is commented. After the optimal experimental conditions were selected, the effect of the variation of the mass and the temperature was explored in order to define the validity limits of the theory. Finally, an investigation was also carried out regarding the thermal unbalance and the subsequent re-equilibration since it affects the experimental HF curve.

\section{Theoretical background}

Among the several geometries available for the development of a theory regarding the evaporation process from a surface, the optimal geometry is by far that of a film characterized by a negligible thickness compared to the other two dimensions (Fig. 1). This geometry ensures a constant surface separating the liquid and the gas phases, avoiding excessive parametrization in the final equation. Thus, a model aiming 


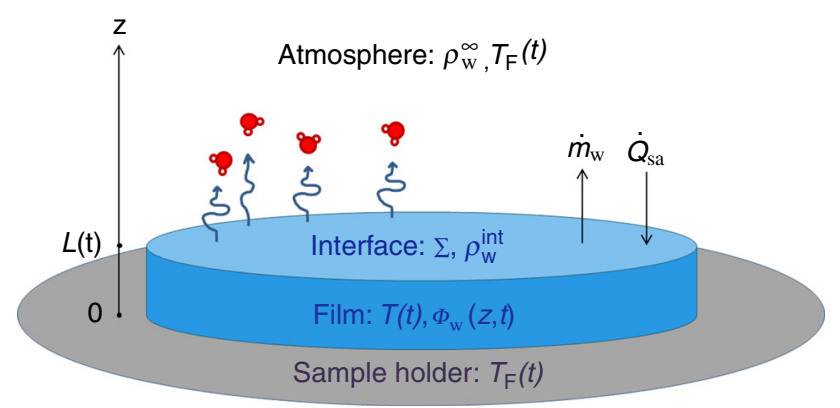

Fig. 1 Schematic representation of the film considered as model system and of the more important parameters

at describing the heat and mass transfer during the isothermal dehydration process of a thin film inside a DSC chamber is here briefly described, while a more detailed dissertation is reported in a previous paper [12].

The rate of convective evaporation from a surface saturated with water can be considered constant if the surface dimension remains constant and all the external conditions are homogeneous and constant (temperature, relative humidity, pressure). Under these circumstances, suitable equations can be developed.

The mass transfer from the aqueous film to the surrounding atmosphere of the DSC chamber is driven by the difference between the vapor density $\rho_{\mathrm{w}}^{\text {int }}$ at the free interface and the $\rho_{\mathrm{w}}^{\infty}$ far from the aqueous film. Thus, the rate of mass transfer is expressed by the following equation:

$\frac{\mathrm{d} m}{\mathrm{~d} t}=\sum_{m} h_{\mathrm{m}}\left(v_{\infty}\right)\left[\rho_{\mathrm{w}}^{\mathrm{int}}\left(T_{\mathrm{int}}\right)-\rho_{\mathrm{w}}^{\infty}\left(T_{\mathrm{F}}\right)\right]=-\sum_{m} \Phi_{\mathrm{m}}$,

where $h_{\mathrm{m}}$ is the coefficient for mass transfer. On the other hand, the heat transfer inside the DSC chamber can be described by the following heat balance equation derived from a simple thermoelectric analogy (Fig. 2):

$$
\begin{aligned}
\left(K+c_{\mathrm{w}} m_{\mathrm{w}}(t)\right) \frac{\mathrm{d} T}{\mathrm{~d} t}= & h_{\mathrm{e}} \sum_{m}\left(T_{\mathrm{F}}(t)-T(t)\right)-\sum_{m} \Phi_{\mathrm{m}} L_{\mathrm{v}} \\
& +\dot{Q}_{\mathrm{sh}},
\end{aligned}
$$

where $h_{e}$ is the heat transfer coefficient, $c_{\mathrm{w}}$ is the liquid water heat capacity, $K$ is a constant that depends on the substrate, $L_{\mathrm{v}}$ is the vaporization heat of water, and $\dot{Q}_{\mathrm{sh}}$ is the conductive heat transfer from the sample holder to the sample, expressed by:

$\dot{Q}_{\mathrm{sh}}=\left(T_{\mathrm{F}}(t)-T(t)\right) / C$,

where $C$ is a constant that depends on the calorimeter.

As reported by Heyd et al. [12], the model above described provides the value of the actual water activity inside the film during the dehydration process as a result of the treatment of the calorimetric experimental data, provided that the quasiequilibrium thermodynamic conditions are saved.

\section{From theory to experiment: setup optimization}

\section{Using a cellulose substrate for a planar geometry}

Since the sample mass is of the order of magnitude of few milligrams (let say about $4 \mathrm{mg}$ ), in order to minimize the possible effect of the surface tension of the water and to simulate a planar geometry, a paper support of circular shape that fits the cavity of the DSC aluminum pan was used. The use of a paper substrate allowed the constancy of the surface between the water and the environment during the whole evaporation process. The average characteristics
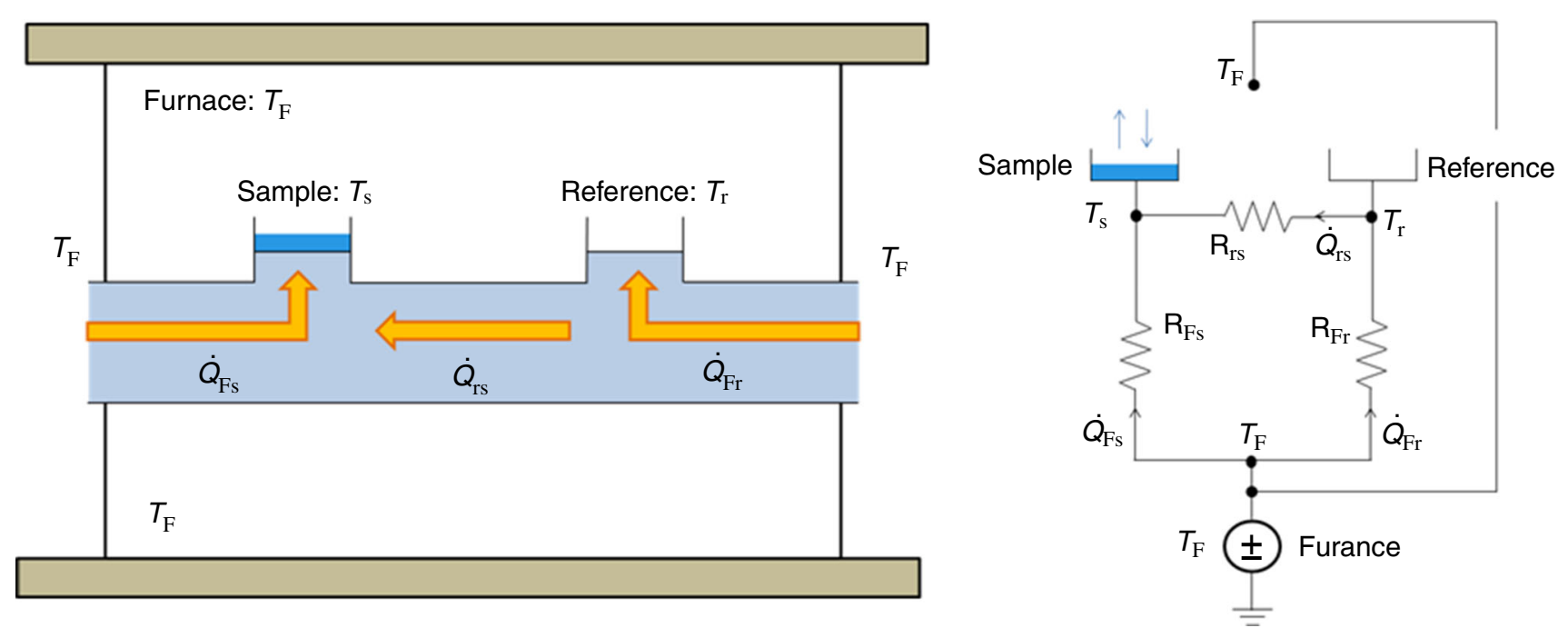

Fig. 2 Schematic representation of the heat flow into the calorimetric chamber (left) and thermoelectric analogy (right) 
of the paper disk were measured over 40 disk samples. The diameter of the paper disk was about $5.9 \mathrm{~mm}$ with an average surface area of $27.3 \mathrm{~mm}^{2}$ and unitary thickness dimension of $0.11 \mathrm{~mm}$; the average mass was $1.6 \pm 0.1 \mathrm{mg}$ in a total gross volume of about $3 \mathrm{~mm}^{3}$. Thus, at least $2 \mathrm{mg}$ of water can fit the macroporous structure of the paper disk; additional water is mainly layered above the upper surface and, in part, between the paper disk and the aluminum pan.

The use of a cellulose substrate implies to take into consideration an additional heat capacity term in the model treatment (assuming a specific heat of $1.17 \mathrm{~J} \mathrm{~g}^{-1}{ }^{\circ} \mathrm{C}^{-1}$ the constant $K$ of Eq. 2 will have an additional contribution of $1.9 \mathrm{~mJ}^{\circ} \mathrm{C}^{-1}$ ) [12]. The actual influence of the dry paper disk on the measurements was evaluated by comparing calorimetric curves with and without a paper disk in the reference pan. Since no changes were detected in the curves, a negligible contribution of the paper substrate on the $K$ constant was assumed. Still, from the practical viewpoint, all standard measurements were taken by placing a paper disk in the reference pan.

Analogously, very slightly different results were obtained by comparing measurements taken using a new paper disk and a paper disk already used for a previous experiment of water evaporation. However, since modifications in the structure of the cellulosic fibers upon complete dehydration are known from the literature [15], a new paper disk was used for each measurement. In conclusion, the effect of water-cellulose interaction on the dehydration process is not discussed here, although this interaction seems to affect the final part of the dehydration curve [16] and will be further investigated taking into account the most recent calorimetric results on nanocellulose fibers [17].

\section{Comparison between the gravimetric and calorimetric mode}

In the crude assumption that the calorimetric response is simply proportional to the rate of mass undergoing evaporation, there should be a correspondence between gravimetric and calorimetric experiments, beside the differences occurring in the setup. Thus, the effect of the sample geometry on water evaporation was investigated by parallel gravimetric and calorimetric experiments at $20^{\circ} \mathrm{C}$, considering the two different geometries, planar surface (water film on paper disk) and spherical surface (water drop). The challenge was to compare the two results and evaluate the performance of a calorimeter, by using the HF instead of the mass as experimental parameter.

A consistent way to present a collection of gravimetric data is to report the mass of water as a function of the time until a lower plateau is reached, which corresponds to the loss of all the water. As reported in Fig. 3a, the gravimetric curve is characterized by an initial straight line in both cases (water evaporation from drop and from film), with a small slowing down of water evaporation in the final part before reaching the plateau. The slope of the linear part represents the rate of water evaporation, in the present experiments 0.09 and $0.15 \mathrm{mg} \mathrm{min}^{-1}$ for the drop and for the film, respectively. The evaporation of a water sample deposited as a drop is slower than the same amount of water deposited as a film on the paper substrate.

The calorimetric results on the same systems (water as drop and as film) are shown in Fig. 3b, where it should be noted that the reported signal (HF) is effectively a measure of the evaporation rate. The typical trend of the HF versus temperature in isothermal conditions is characterized by an initial part affected by the calorimetric unbalance and subsequent re-equilibration (see "Thermal unbalance and re-equilibration" section), followed by the "quasi-constant" evaporation rate segment and the final sigmoidal decay. The evaporation from the drop is slower than that from the film; the shape of the two calorimetric curves is quite different, especially in the final part, where the sigmoidal decay of the sample on paper is smoother than that of the drop sample. This behavior indicates that on approaching the end of the evaporation, the phenomena due to the interaction of water with cellulose substrate are detectable. For a better comparison with gravimetric data, Fig. $3 \mathrm{c}$ reports the integral of the HF curves of Fig. 3b. By using this mathematical approach of integration, it is possible to mimic the water mass loss in the same way as it is obtained from the direct gravimetric measurements. The slope of the linear part, which represents the rate of water evaporation, was 0.08 and $0.10 \mathrm{mg} \mathrm{min}^{-1}$ for the drop and for the film, respectively.

The experimental data only in part confirm the assumption based on surface considerations, even though the actual evaporation rates are affected by many time-dependent changes. In the case of the paper support, water can be partially entrapped in the large pores of the paper disk, thus reducing its effective evaporation rate with time, while in the water drop, the effect of the contact angle and the deviation from spherical volume during the evaporation process should be taken into account. Indeed, the surface calculated at time zero is $12.1 \mathrm{~mm}^{2}$ for the sphere and $27.3 \mathrm{~mm}^{2}$ for the disk, respectively. Since the sphere is not floating, its actual surface is expected to deviate from that calculated for the suspended drop; analogously, the effective surface of the paper disk may be affected by the cellulose solid network.

It has to be noticed that while in the case of the paper, the curves obtained with either gravimetric or calorimetric measurements have similar shapes, and in the case of the drop, a difference is observed at the end of the process. Indeed, the gravimetric curve for a drop tends to a plateau in a smooth manner, while the integral curve corresponding 

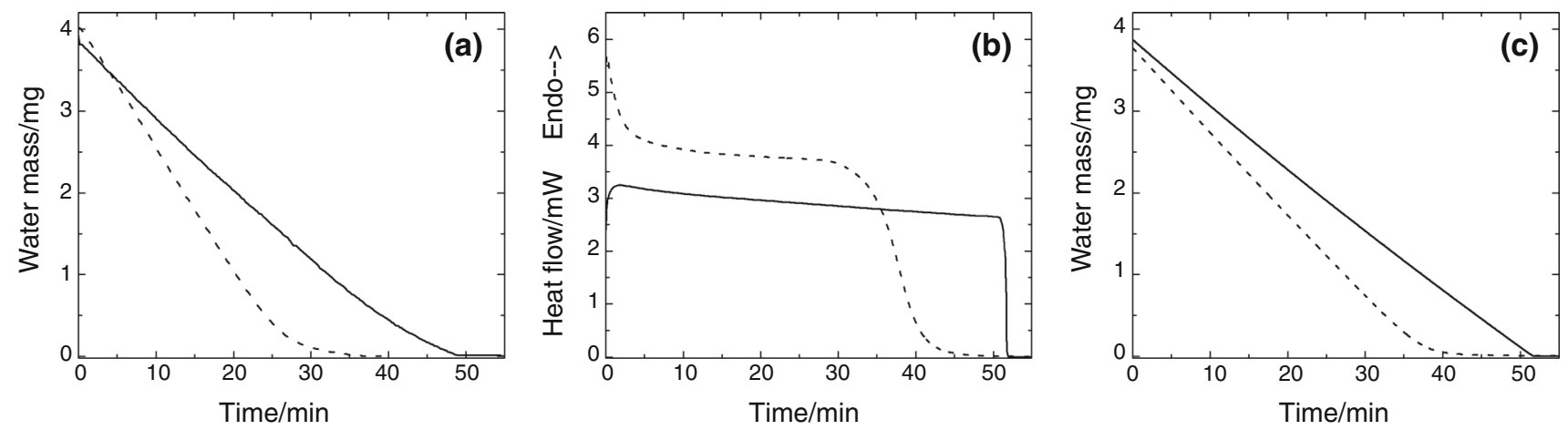

Fig. 3 Water evaporation in isothermal conditions at $20{ }^{\circ} \mathrm{C}$ from two different geometry samples: dashed lines refer to film on paper disk, solid lines to drop. a Gravimetric data at RH $40 \%, 3.92 \mathrm{mg}$ of water

to water as a drop is characterized by a net change of the slope before reaching the plateau.

In principle, both gravimetric and calorimetric experiments give information about the rate of mass loss during the evaporation that in the calorimetry can be obtained from the HF partial integration. It is worth noticing that calorimetry is more sensitive than gravimetry to small mass differences. This is of particular interest for samples with different composition, since differences in the evaporation appear more evident in the final part of the evaporation process, as already discussed in a previous paper [12, 18].

Thus, despite many concordant similarities between gravimetric and calorimetric experiments, the differences both quantitative and qualitative in the experimental curves can be amply justified by the differences in the two setups due, in particular, to the geometry of the chambers and to the gas flow rate.

\section{Nitrogen flux}

The theoretical model developed in the "Theoretical background" section is based on the assumption that the vapor pressure in the surrounding environment is approximately zero. This condition is easily reached in practice by setting a suitable nitrogen flux that can guarantee a negligible relative humidity inside the DSC chamber. Since very high purging gas fluxes introduce many counteractions, the effect of different nitrogen flux on the rate of water evaporation was investigated by changing the output pressure from the nitrogen tank. High values of nitrogen flux can generate turbulent motions inside the DSC chamber, and thus, the measure and the theoretical modeling of the evaporation process would be compromised. On the other hand, low evaporation rates do not guarantee a negligible vapor pressure inside the DSC chamber. as drop and $4.01 \mathrm{mg}$ of water on paper, b calorimetric data, $3.87 \mathrm{mg}$ of water as drop and $3.77 \mathrm{mg}$ of water on paper, $\mathbf{c}$ integral of curves of (b)

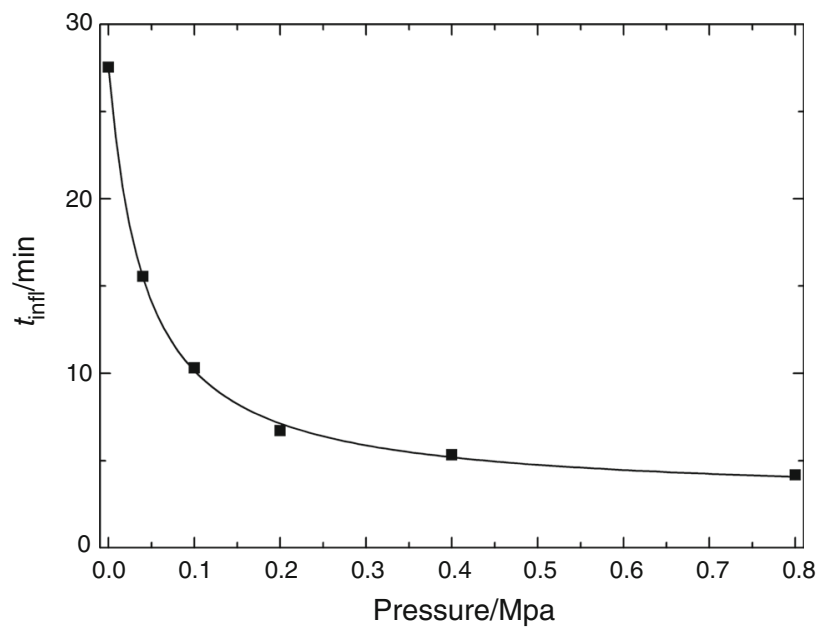

Fig. 4 Inflection time for a sample of water $(8 \mathrm{mg})$ deposited on a paper disk, reported as a function of the nitrogen pressure (output gas cylinder)

Figure 4 reports the parameter inflection time $\left(t_{\text {infl }}\right)$ as a function of the gas pressure for a sample of water $(8 \mathrm{mg})$ deposited on a paper disk. The parameter $t_{\text {infl }}$ is defined by the inflection point of the sigmoidal HF curve and gives an estimate of the total evaporation time. From the experimental data of Fig. 4, the range between 0.1 and $0.2 \mathrm{MPa}$ can be considered a good compromise between high and low evaporation rates, and a pressure of 1 bar was selected for the experiments, which corresponds to a nitrogen flux of about $15 \mathrm{~mL} \mathrm{~min}^{-1}$.

\section{Sample mass and temperature}

\section{Sample mass}

The effect of the initial sample mass was mainly investigated at $20{ }^{\circ} \mathrm{C}$ under controlled conditions of all the other external parameters. The comparison of the curves 
obtained with different mass required to identify some characteristic parameters that take into account both thermodynamic (enthalpy) and kinetic (evaporation rate) aspects of the evaporation process. For the first point, the integral of the HF versus time can be evaluated from the curve and normalized by the water mass to provide $\Delta H_{\mathrm{vap}}$; for the second point, the question concerns the definition of an appropriate parameter that refers to the total time of the evaporation process. Indeed, the evaporation rate is a continuous function of the time and the experimental HF curves are characterized by a quasi-constant rate until the final decay. Since the final evaporation time cannot be accurately identified at the lower plateau of the curve when the evaporation process is completed, the inflection point $\left(t_{\text {infl }}\right)$ of the sigmoidal curve was contemplated as a suitable parameter. This choice appeared simple and practical, and less affected by possible experimental errors.

As an example, Fig. 5a reports a series of measurements taken at $20{ }^{\circ} \mathrm{C}$, where the $\mathrm{HF}$ is reported as a function of the initial mass. The same curves, where the time axis of each curves was normalized by the corresponding value of $t_{\text {infl }}$, are reported in Fig. 5b. According to this approach, a common behavior in the "quasi-constant range" and a remarkable superimposition of the tail of the curves was found. In addition, this approach allowed a valuable comparison among curves, pointing out the differences for the samples with lower water mass.

Figure 5c shows the values of $\Delta H_{\text {vap }}$ and $t_{\mathrm{n}}$ reported as a function of water mass. A linear correlation was found between the water mass and the $t_{\text {infl. }}$ The slope is the $t_{\mathrm{n}}$ parameter that is characteristic for a set of measurements on the same sample (here water). Constant values for both the parameters chosen were evidenced, thus confirming the validity of the choice and the reproducibility of the evaporation process. In particular, for pure water, $\Delta H_{\text {vap }}$ is $2.28 \pm 0.04 \mathrm{~kJ} \mathrm{~g}^{-1}$ and $t_{\mathrm{n}}$ is $10.1 \pm 0.1 \mathrm{~min} \mathrm{mg}^{-1}$, at
$20{ }^{\circ} \mathrm{C}$ [12]. However, the experimental $\Delta H_{\text {vap }}$ was found to be lower than the average value only for the lowest mass of water. This behavior is mainly attributed to the fact that the evaporation starts before the insertion of the sample inside the DSC chamber and to the thermal unbalance after the sample is inserted; the latter represents the major source of error.

In conclusion, the reproducibility and the validity of the experiments in isothermal conditions were investigated by using the heat of evaporation $\Delta H_{\text {vap }}$ and the evaporation time $t_{\mathrm{n}}$ defined as the inflection point $t_{\text {infl }}$ normalized by the sample mass.

\section{Temperature}

In order to trace the range of validity for the isothermal calorimetric measurements, the curves of water evaporation from 10 to $100{ }^{\circ} \mathrm{C}$ were investigated (Fig. 6a). All the curves display the already discussed behavior characterized by a quasi-constant range followed by a rapid decrease in the HF when approaching the complete dehydration. Upon increasing the temperature, an increase in the evaporation rate in the quasi-constant regime is observed, and as a consequence, the evaporation time is shortened. Before analyzing the parameters, it is worth pointing out that the "quasi-constant range" shows values of HF (i.e., the calorimetric $\Delta T$ ) that increases with the temperature; furthermore, curves carried out at temperature higher than $50{ }^{\circ} \mathrm{C}$ were characterized by changes in the slope of the "quasi-constant range." Both these experimental results do not satisfy the theoretical assumptions of the model and have to be discussed [12].

The normalization of the time with the parameter $t_{\text {infl }}$ still allows to compare curves carried out in a wide range of temperature (Fig. 6b). It is worth noticing that the initial
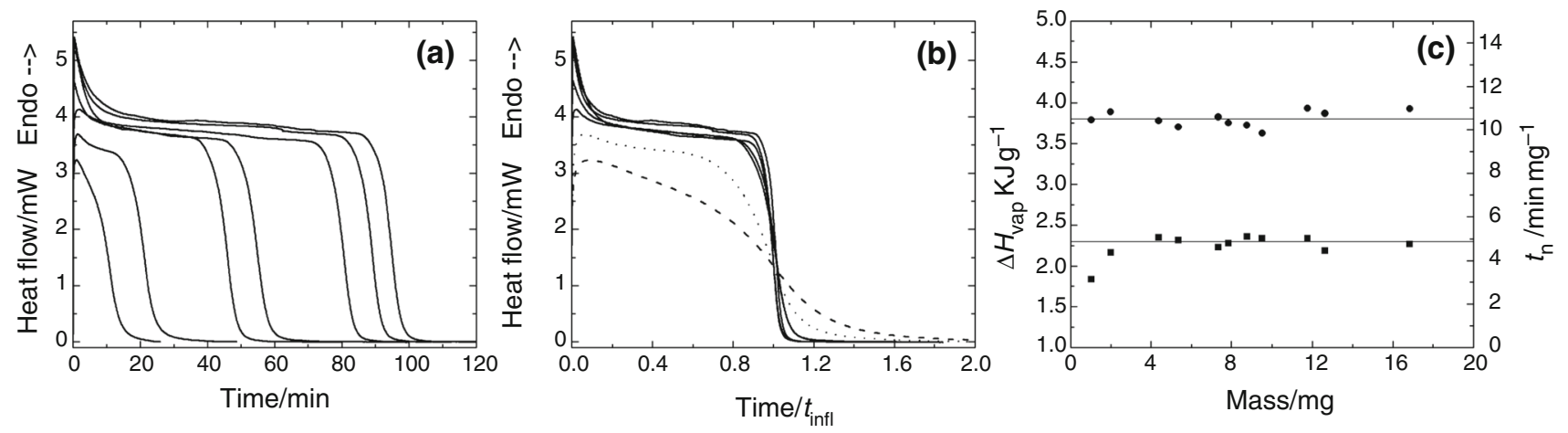

Fig. 5 a Calorimetric curves of water evaporation at $20{ }^{\circ} \mathrm{C}$ from film on paper disk as a function of the sample mass: 1.03, 1.99, 4.37, 5.36, $7.84,8.75,9.52 \mathrm{mg}$ (respectively from left to right). b Curves of Fig. $4 \mathrm{a}$ where the time is normalized by the parameter $t_{\text {infl }}: 1.03 \mathrm{mg}$

(dash), $1.99 \mathrm{mg}$ (dot), 4.37-9.52 mg (solid). c Enthalpy of evaporation $\Delta H_{\text {vap }}$ (filled square) and $t_{\mathrm{n}}$ (filled circle) as a function of the sample mass 

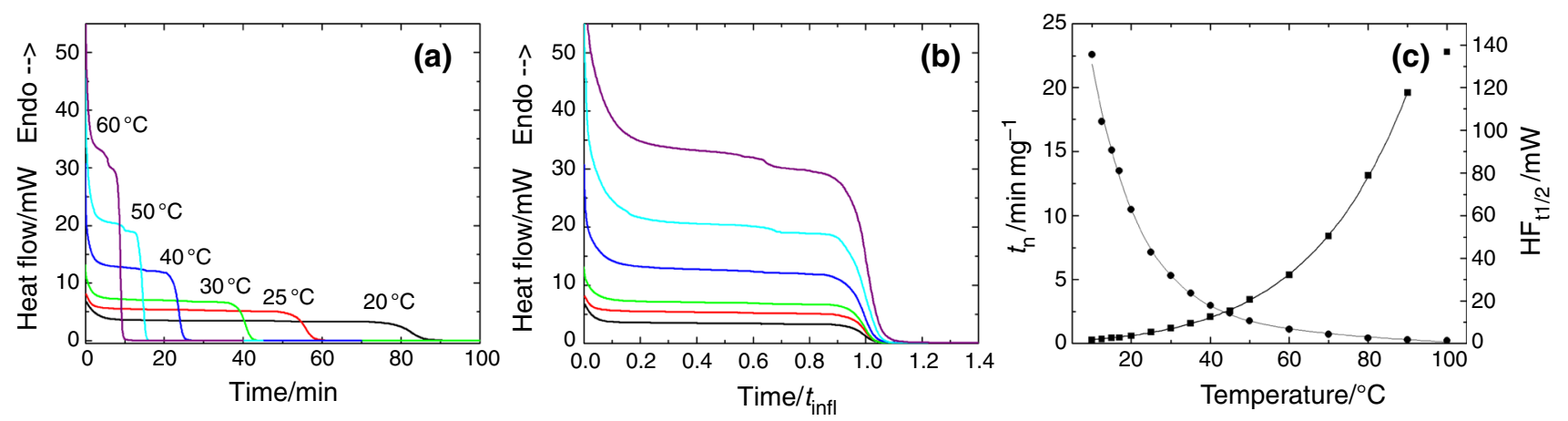

Fig. 6 Curves of water samples at different temperature $\mathbf{a}$ as a function of time and $\mathbf{b}$ as a function of the time normalized by the $t_{\text {infl }}, \mathbf{c}$ values of $\mathrm{HF}_{\mathrm{t} 1 / 2}$ (filled square) and $t_{\mathrm{n}}$ (filled circle) reported as a function of the temperature

thermal unbalance and re-equilibration is more evident as higher is the difference between the external environment and the calorimetric chamber, since the samples were always prepared at $20^{\circ} \mathrm{C}$.

For each curve, the parameter $t_{\mathrm{n}}$ was calculated in addition to the parameter $\mathrm{HF}_{\mathrm{t} 1 / 2}$, corresponding to the $\mathrm{HF}$ at one half of the $t_{\text {infl }}\left(t_{1 / 2}\right)$. Both are reported in Fig. 6c, as a function of the temperature. The change of these two parameters as a function of the temperature follows an exponential law. An increase in $\mathrm{HF}_{\mathrm{t} 1 / 2}$ is counterbalanced by the decrease in the $t_{\mathrm{n}}$.

Thus, $\Delta H_{\text {vap }}$ was calculated with two procedures: the first one as the total area under the curve (integral area), as already reported in Fig. 5c for the effect of the mass of the sample; the second one as the product between the values $\mathrm{HF}_{\mathrm{t} 1 / 2}$ and $t_{\mathrm{n}}$. The experimental values of $\Delta H_{\mathrm{vap}}$ are systematically lower than the literature values [19] with a deviation of about $10 \%$ and even more at temperatures higher than ca. $70{ }^{\circ} \mathrm{C}$ (see Fig. 7).

Figure 7 also reports the data of $\Delta H_{\text {vap }}$ corrected according to the procedure outlined in "Thermal unbalance and re-equilibration" section, which includes some corrections at the very beginning of the measurement. The effect of this contribution is particularly relevant upon increasing temperature, although only the trend is improved, while the absolute values still remain lower than the literature values. These findings clearly evidenced that, in order to get reliable results of heat of evaporation from isothermal measurements, some other corrections are required for the current experimental setup.

\section{Thermal unbalance and re-equilibration}

Although the results above reported consistently show a clear quantitative correlation between the calorimetric results and the operational parameters, still some aspects reflect the intrinsic problems that calorimetric measurements imply in the analysis of the data. The purpose of this

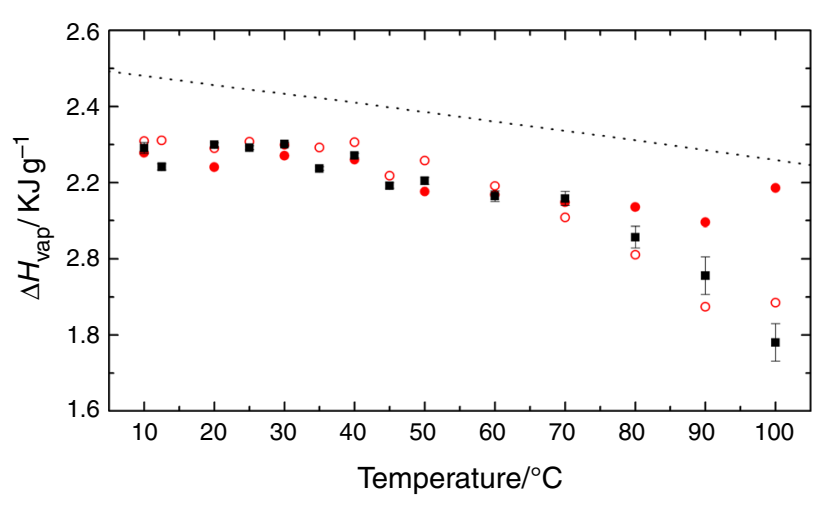

Fig. 7 Evaporation enthalpy for water $(8 \mathrm{mg}$ ) deposited on a paper disk, calculated as the product between $\mathrm{HF}_{\mathrm{t} 1 / 2}$ and $t_{\mathrm{n}}$ (filled square), integral area of the calorimetric curve (open circle) and integral area corrected for the contributions $A_{1}, A_{2}$ and $A_{3}$ (filled circle). Dotted line refers to the literature values [19]

section is to analyze the specific influence of the thermal unbalance on the initial record of the signal and to exploit alternative experimental procedures.

\section{Equilibration time of the calorimeter}

In the common experimental procedure, the sample is inserted in the calorimetric chamber, and then the measure is started only after a sufficient time for the equilibration of the system (standard procedure). This is controlled by the constancy of the HF as the signal measured with the thermocouple is proportional to the temperature difference between the sample and the reference. In the present experimental procedure, the sample is weighed and transferred to the calorimeter and the measure is immediately started, with a time lapse of $10 \mathrm{~s}$. Thus, even if the measurement is in isothermal modality, the initial part of the recorded curve shows the tail of the thermal unbalance and introduces some errors in both the correct HF value and the correct time of the evaporation process. 
Alternatively, if the measurement could be continuously recorded, the thermal unbalance would be recorded as a peak at the time $t_{\mathrm{o}}$ of the insertion of the sample in the calorimeter, still affecting the initial part of the measurement and, therefore, introducing a bias. To quantify the entity of the signal due to the unbalance and the subsequent re-equilibration of the calorimeter, this issue was approached by recording the measurement before the sample was inserted (modified procedure). As an example, Fig. 8 shows the effect of the calorimetric unbalance and subsequent re-equilibration obtained at $70{ }^{\circ} \mathrm{C}$ after the insertion of a sample into the chamber; the two curves refer to a pan with water deposited on a paper disk and to a blank with a paper disk only. The difference between the two curves shows clearly the signal effectively due to the evaporation.

In order to describe mathematically the shape of these curves and the parameters involved, it is necessary to resort to the basic equations developed for the conduction calorimeters [20-23].

\section{Calorimeter response to the thermal unbalance}

Referring to the two experiments shown in Fig. 8, the transient response of a thermocouple to a temperature change of the calorimetric cell is governed by the following differential equation:

$\tau \frac{\mathrm{d} y}{\mathrm{~d} t}+y=k \cdot F(t)$

where $F(t)$ is the external stress (heat capacity of the sample inserted into the calorimetric chamber) and $y(t)$ is the response of the calorimeter.

In the specific case the system consists of a paper substrate (constant heat capacity), the stress function is:

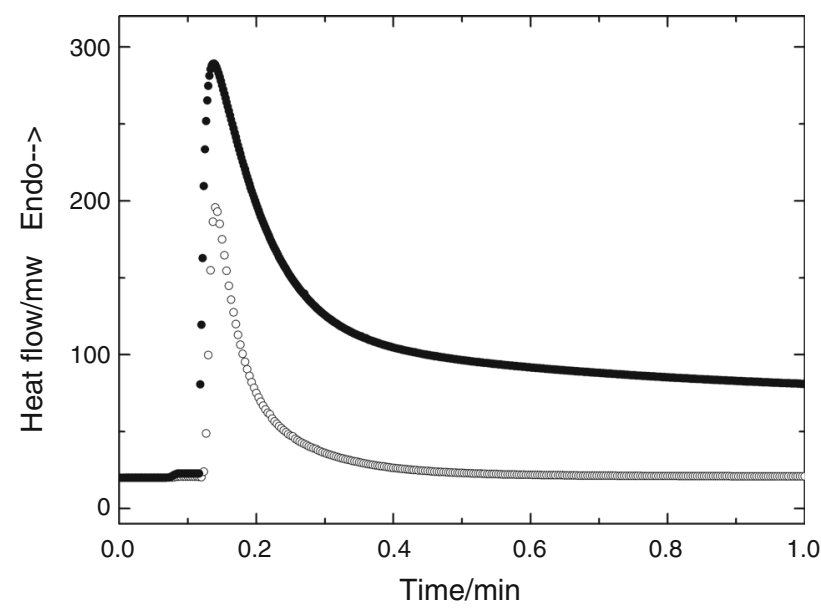

Fig. 8 Calorimetric curves (modified procedure) recorded at $70{ }^{\circ} \mathrm{C}$ for water $(8 \mathrm{mg})$ deposited on paper disk (filled circle) and for paper disk alone (white circle)
$F(t)=\left\{\begin{array}{cc}0, & t<t_{0} \\ A, & t \geq t_{0}\end{array}\right.$

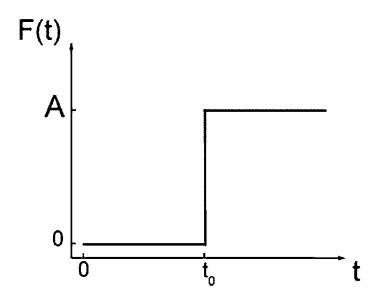

where $A$ can be taken constant in a small temperature range and the solution of the problem is:

$y(t)=C_{1}+C_{2} \mathrm{e}^{-\left(\mathrm{t}-\mathrm{t}_{0}\right) / \tau}$,

where

$$
\left\{\begin{array}{l}
C_{1}=k A \\
C_{2}=y_{0}-A
\end{array}\right.
$$

In the case of water sample deposited on the paper substrate (heat capacity decreasing with the time), the stress function is:

$F(t)= \begin{cases}0, & t<t_{0} \\ A-b \cdot\left(t-t_{0}\right), & t \geq t_{0}\end{cases}$

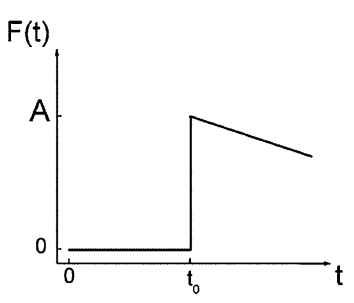

where both $A$ and $b$ depend on the temperature of the isotherm, and the solution of the problem is:

$y(t)=C_{1}+C_{2} \mathrm{e}^{-\left(\mathrm{t}-\mathrm{t}_{0}\right) / \tau}+C_{3} \cdot\left(t-t_{0}\right)$,

where

$$
\left\{\begin{array}{l}
C_{1}=k(A+b \tau) \\
C_{2}=y_{0}-k\left[A+b\left(\tau-2 t_{0}\right)\right] \\
C_{3}=-k b
\end{array}\right.
$$

Therefore, two temporal parameters $t_{0}$ and $\tau$, characteristic of the first stage of the measurement, can be defined. The first parameter $t_{0}$ corresponds to the actual insertion of the sample into the calorimeter that gives the impulse of unbalancing; it is a delay time with respect to the beginning of signal recording and depends on the operator. The second parameter $\tau$ is the characteristic time of response of the calorimetry, and it is the time necessary for approaching the re-equilibration (usually considered to 
occur at $5 \tau$, corresponding to the $99.3 \%$ of the reequilibration).

Using Eqs. 6 and 9, the parameter $\tau$ was calculated for all the isothermal measurements. Since no dependence of $\tau$ was found with temperature, the value of $\tau$ was averaged on the overall measurements at different temperatures and resulted to be $3.4 \mathrm{~s}$ for the paper disk and $4.8 \mathrm{~s}$ for $8 \mathrm{mg}$ of water on the paper disk. In addition, the mean value of $t_{0}$ was found to be $8 \mathrm{~s}$. It is worth noticing that shorter characteristic time of $0.4 \mathrm{~s}$ is necessary in order to fit the whole curve.

\section{Evaluation of the evaporation enthalpy}

In "Sample mass and temperature" section, the effect of mass and temperature on the shape of curves has been presented. The enthalpy associated with the evaporation process as a function of water mass and temperature has been calculated by taking the area under the experimental curve (Figs. 5a, 6a), while the mass is taken as the mass difference before and after the evaporation. These data are shown in Figs. 5c and 7; in the last figure, as an apparently rough approximation, the area of the equivalent rectangular figure having the dimension of $t_{\mathrm{n}}$ and $\mathrm{HF}_{\mathrm{t} 1 / 2}$ was also evaluated.

As clearly shown in Fig. 8, the initial part of the calorimetric curve for the isothermal evaporation at the temperature $T$ is affected by two issues: (a) a delay $t_{0}$ occurring between the weighing the water deposited on the paper disk and the beginning of the signal recording and (b) the unbalance and the subsequent re-equilibration of the calorimeter after the insertion of the sample into the chamber (abrupt temperature and heat capacity changes). The aim here is to estimate these missing parts of the HF

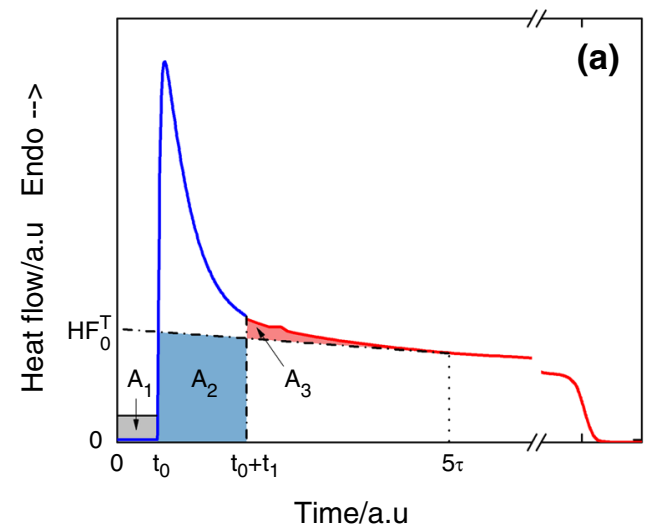

Fig. 9 a curves of water evaporation ( $8 \mathrm{mg}$ ) using the standard (red) and the "modified" (blue) procedure; contributions $A_{1}, A_{2}$ and $A_{3}$ are evidenced (note that $x$ axis has been magnified and that in a real signal recorded in the standard modality in order to evaluate the error affecting the calculation of $\Delta H_{\mathrm{vap}}$. This issue was pursued by comparing the standard procedure with the modified procedure that contains all the information on the unbalance (Fig. 9).

If no instrumental unbalance occurs and the calorimetric signal is only due to the heat capacity variation of the sample, a linear behavior of the curve would be expected. By a simple linear fitting of the quasi-constant part, the initial part of the curve (from 0 to $t_{0}+5 \tau$ ) could be reconstructed (dot-dash line in Fig. 9a).

1. The area $A_{1}$ corresponds to the fraction of water mass evaporating at room temperature $\left(20^{\circ} \mathrm{C}\right)$ in the timeframe between the weighing operation and the insertion in the calorimeter. Under controlled laboratory operation, this contribution is a fixed amount and can be estimated as the product of $t_{0}$ times $\mathrm{HF}_{0}$ (at $20{ }^{\circ} \mathrm{C}$ ). The area of the contribution is invariant with the temperature of calorimetric experiment and is about $32 \mathrm{~mJ}$ for the evaporation of a small fraction water, corresponding to an enthalpy contribution $4 \mathrm{~J} \mathrm{~g}^{-1}$ to the total change of $2300 \mathrm{~J} \mathrm{~g}^{-1}$.

2. The area $A_{2}$ corresponds to the fraction of evaporation at the temperature $T$ lost in the time frame (time $t_{1}$ ) in which the initial calorimetric unbalance has to be reequilibrated and the recording has not started yet. The contribution $A_{2}$ was calculated from the curve (at $T$ ) as the product between $t_{1}$ and $\mathrm{HF}_{0}$ and increases with the temperature.

3. The area $A_{3}$ under the experimental curve and above the fitting line is due to a residual thermal unbalance and does not correspond to a change associated with the evaporation of the sample, and thus, it has to be subtracted from the integral area under the curve.

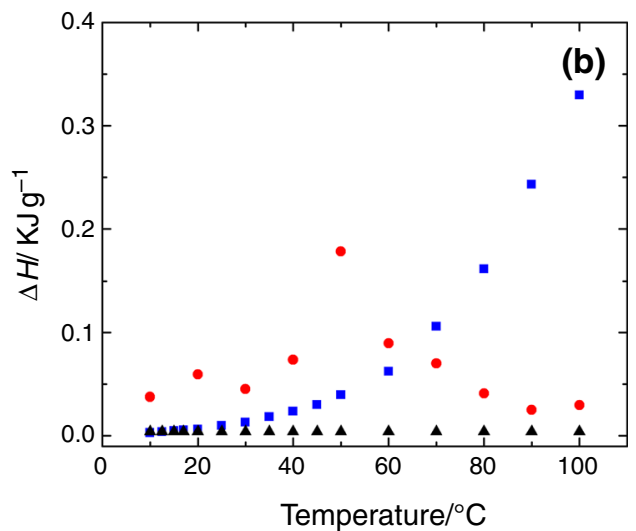

experiment the evaporation time $t_{\text {infl }}$ can be as large as 1000 times the value of $\tau$ ). b $A_{1}$ (filled triangle), $A_{2}$ (filled square) and $A_{3}$ (filled circle) are reported as a function of the temperature 
Thus, the contributions $A_{1}, A_{2}$ and $A_{3}$ were evaluated (Fig. 9b) and their temperature dependence taken into account.

The corrected value of $\Delta H_{\text {vap }}$ is, therefore, given by adding the three contributions $A_{1}, A_{2}$ and $A_{3}$ to the $\Delta H_{\text {vap }}$ value calculated by the simple integration of the calorimetric curve (HF vs time) that are reported in Fig. 7:

$\Delta H_{\text {vap }}^{\text {corrected }}=\Delta H_{\text {vap }}^{\text {integral }}+A_{1}+A_{2}-A_{3}$

As above discussed, the correction only affects the values at high temperature.

\section{Future perspectives and conclusions}

The present calorimetric analysis allows a good control of the environmental temperature and humidity, resulting a suitable approach to study the kinetics of water evaporation from several systems. In order to validate the assumption made for the development of the theoretical model, the influence of some critical parameters was investigated. It is, however, critical for the experiment that water availability in the evaporation process is always under a stationary state, if not at complete equilibrium. These conditions are reached provided that migration of water in the moistened film is not hampered either by the mechanical hardening of the material (i.e., by reaching the glass transition in a isothermal mode) or by the formation of a coating layer depressing the evaporation. In both cases, the equilibration time may become longer than some second, and therefore, the measured HF decreases as a convolution of thermodynamic and kinetic effects. A practical example of such phenomena has been recently given in the analysis of the evaporation of solvent from sugar or salt matrices [1, 4]. However, whenever the structural constraints hamper the free diffusion of the water, the resulting deviation from the thermodynamic condition provides another useful information. Thus, the following conclusions can be drawn from the isothermal DSC study of the dehydration process. By using a hybrid approach, which takes into account both the heat and mass diffusive transfers inside the sample and the heat transfers inside the DSC chamber, it is possible to study the drying process of aqueous solutions without resorting to gravimetric measurements. The very accurate measurement of the heat flux accessible by using a DSC allows for a realistic description of the drying process of complex solutions frequently used in biophysics, as such has been reached by Schiraldi and Fessas [24].

From a mere analytical view point, the study of water evaporation in isothermal mode has the practical advantage that the rate of water evaporation is simply described as the change of $\mathrm{HF}$ as a function of the time. It has been pointed out that under quasi-equilibrium conditions, the experimental data of water evaporation from aqueous solutions can be easily converted in activity curves, provided that the rate of solute migration in the film is comparable with the rate of solvent evaporation. Indeed, in the previous paper, a family of evaporation curves from several sugar solutions has been regarded as a series of moisture desorption isotherms, showing that, even for the low-moisture region, a lower activity is obtained by increasing sugar concentration.

The data here presented give further strength to these basic concepts, although clear limitations and corrections to this approach arise when the isothermal dehydration occurs at high temperature. Therefore, a straightforward use of the same formalism in the investigation of water dehydration in the scanning mode is not possible at this stage, although the scanning curves of aqueous polymer solutions and polymer gels previously published $[25,26]$ effectively follow the trend of the HF curves as a function of temperature, as reported in Fig. 6c. Thus, the correlation between a series of dehydration isotherms as function of temperature and the single dehydration curve in temperature scanning mode is worth to be explored, as well as the comparison of the values of bound water from evaporation experiments with those obtained from freezing/melting curves [27].

Acknowledgements The authors thank A. Rampino and E. Gurian for some preliminary measurements. This work has been partially carried out within the project "Physical-Chemistry of Nanostructured Biomolecular Glasses" supported by Bracco Imaging SpA.

\section{References}

1. Zobrist B, Soonsin V, Luo BP, Krieger UK, Marcolli C, Peter T, Koop T. Ultra-slow water diffusion in aqueous sucrose glasses. Phys Chem Chem Phys. 2011;13:3514-26.

2. Tong H-J, Reid JP, Bones DL, Luo BP, Krieger UK. Measurements of the timescales for the mass transfer of water in glassy aerosol at low relative humidity and ambient temperature. Atmos Chem Phys. 2011;11:4739-54.

3. Amer MS, Wang W. Effect of fullerene nanospheres on water evaporation kinetics and first-order thermal transitions. J Phys Chem C. 2012;116:8216-22.

4. Davies JF, Haddrell AE, Miles REH, Bull CR, Reid JP. Bulk, surface, and gas-phase limited water transport in aerosol. J Phys Chem A. 2012;116(10987):10998.

5. Li K-K, Wang F, Zeng G, Reid JP, Zhang Y-H. Probing the time scale for bulk equilibration and mass transport of water in amorphous inorganic aerosol. J Phys Chem B. 2011;115:14397403.

6. Bones DL, Reid JP, Lienhard DM, Krieger UK. Proc Natl Acad Sci USA. 2012;109:11613-8.

7. Guerrier B, Bouchard C, Allain C, Bénard C. Drying kinetics of polymer films. AIChE J. 1998;44:791-8. 
8. Cevc G, Kornyshev AA. Simple-model of dehydration transitions in lamellar systems, such as lipid-membranes. J Chem Phys. 1993;98:5701-12.

9. Berk Z. Dehydration. In: Food process engineering and technology. 1st ed. New York; 2009. p. 459-510 [chapter 22].

10. Fichot J, Heyd R, Josserand C, Chourpa I, Gombart E, Tranchant JF, Saboungi M-L. Patterned surfaces in the drying of films composed of water, polymer, and alcohol. Phys Rev E. 2012;86: 061601.

11. Sekine Y, Ikeda-Fukazawa T. Structural changes of water in a hydrogel during dehydration. J Chem Phys. 2009;130:034501.

12. Heyd R, Rampino A, Bellich B, Elisei E, Cesàro A, Saboungi M-L. Isothermal dehydration of thin films of water and sugar solutions. J Chem Phys. 2014;140:124701.

13. Kolb CE, Cox RA, Abbatt JPD, Ammann M, Davis EJ, Donaldson DJ, Garrett BC, George C, Griffiths PT, Hanson DR, Kulmala M, McFiggans G, Poschl U, Riipinen I, Rossi MJ, Rudich Y, Wagner PE, Winkler PM, Worsnop DR, O'Dowd CD. An overview of current issues in the uptake of atmospheric trace gases by aerosols and clouds. Atmos Chem Phys. 2010;10: 10561-605.

14. Whitaker S. Simultaneous heat, mass, and momentum transfer in porous media: a theory of drying. In: Hartnett JP, Irvine TF, editors. Advances in heat transfer, vol. 13. New York: Academic Press, Inc.; 1977. p. 119-203.

15. Park S, Venditti RA, Jameel H, Pawlak JJ. Changes in pore size distribution during the drying of cellulose fibers as measured by differential scanning calorimetry. Carbohydr Polym. 2006;66: 97-103.

16. Park S, Venditti RA, Jameel H, Pawlak JJ. Studies of the heat of vaporization of water associated with cellulose fibers characterized by thermal analysis. Cellulose. 2007;14:195-204.
17. Hatakeyama T, Inui Y, Iijima M, Hatakeyama H. Bound water restrained by nanocellulose fibres. J Therm Anal Calorim. 2013;113:1019-25.

18. Park S, Venditti RA, Jameel H, Pawlak JJ. Hard to remove water in cellulose fibers characterized by high resolution thermogravimetric analysis-methods development. Cellulose. 2006;13: 23-30.

19. Wagner W, Pruß A. The IAPWS formulation 1995 for the thermodynamic properties of ordinary water substance for general and scientific use. J Phys Chem Ref Data. 2002;31:387-535.

20. Calvet E, Prat H. Recent progress in microcalorimetry. New York: Pergamon Press; 1963. p. 30-44.

21. Randzio SL, Suurkuusk J. Interpretation of calorimetric thermograms and their dynamic corrections. In: Beezer AE, editor. Biological microcalorimetry. London: Academic Press; 1980. p. 311-41.

22. Holman JP. First order systems. In: Experimental methods for engineers, 7th ed. New York: McGraw-Hill; 2001. p. 19-23.

23. Holman JP. Thermocouples. In: Experimental methods for engineers, 7th ed. New York: McGraw-Hill; 2001. p. 368-377.

24. Schiraldi A, Fessas D. Classical and Knudsen thermogravimetry to check states and displacements of water in food systems. J Therm Anal Calorim. 2003;71:225-35.

25. Bellich B, Borgogna M, Cok M, Cesaro A. Water evaporation from gel beads. A calorimetric approach to hydrogel matrix release properties. J Therm Anal Calorim. 2011;103:81-8.

26. Bellich $\mathrm{B}$, Borgogna $\mathrm{M}, \mathrm{Cok} \mathrm{M}$, Cesàro $\mathrm{A}$. Release properties of hydrogels: water evaporation from alginate gel beads. Food Biophys. 2011;6:259-66.

27. Mlčoch T, Kučerík J. Hydration and drying of various polysaccharides studied using DSC. J Therm Anal Calorim. 2013;113: $1177-85$. 